\title{
Dynamical Reference Frame - Current Relevance and Future Prospects
}

\author{
E. M. Standish Jr. \\ Jet Propulsion Laboratory; California Institute of Technology, JPL \\ 301-150; Pasadena, CA 91011
}

\begin{abstract}
Planetary and lunar ephemerides are no longer used for the determination of inertial space. Instead, the new fundamental reference frame, the ICRF, is inherently less susceptible to extraneous, non-inertial rotations than would be a dynamical reference frame determined by the ephemerides. Consequently, the ephemerides are now adjusted onto the ICRF, and they are fit to two modern, accurate observational data types: ranging (radar, lunar laser, spacecraft) and VLBI (of spacecraft near planets).

The uncertainties remaining in the inner planet ephemerides are on the order of 1 kilometer, both in relative positions between the bodies and in the orientation of the inner system as a whole. The predictive capabilities of the inner planet ephemerides are limited by the uncertainties in the masses of many asteroids. For this reason, future improvements to the ephemerides must await determinations of many asteroid masses. Until then, it will be necessary to constantly update the ephemerides with a continuous supply of observational data.
\end{abstract}

\section{Introduction}

A dynamical reference frame is inherent within a given set of ephemerides, and the accuracy of the frame depends directly upon the accuracies of the ephemerides themselves. In the past, the ephemerides and their reference frame played a significant role in the determination of inertial space: the motions of the Sun, Moon, and planets were used to eliminate rotations from existing star catalogs.

The situation has changed. The recently adopted IAU International Celestial Reference Frame (ICRF) is assumed to be free from rotation; that is, any systematic motion of the ICRF radio sources is assumed to be negligible for present-day considerations. Further, modern ephemerides are automatically adjusted onto the ICRF through the use of ICRF-based VLBI measurements of spacecraft near planets. Similarly, catalogs in various wavelengths are adjusted onto the system of the ICRS. As such, the dynamical reference frame is no longer used for the determination of non-rotating reference frames.

This paper discusses the creation of modern ephemerides and how the various ephemeris quantities are determined by modern observational data. The orientation of the ephemerides onto the ICRF is shown, and the accuracies of 
the various ephemeris quantities are discussed. Finally, it is mentioned that the concepts of ecliptic, obliquity, and mean equator are now obsolete in the context of modern ephemeris creation.

\section{Ephemerides Determine Inertial Space}

The motions of the Sun, Moon, and planets were used at one time to indicate extraneous rotations embedded within the stellar catalogs. This is a consequence of the fact that ephemeris motion, modeled with realistic equations of motion and fit to observational data, cannot permit a spurious rotation with respect to the reference system. As a consequence, the ephemerides have acted as indicators of systematic errors in the proper motions of star catalogs. Certainly, if the ephemerides were not based upon realistic equations of motion, but were the result of only curve-fitting instead, then the motions of the orbital planes and the ellipses would not be constrained by physics and the inertial basis would be lost.

This may be most easily envisaged for a one-body solar system: the body moves in a straight line with constant velocity. Since the equations provide for only straight-line motion, the ephemeris of the body is automatically cast in an inertial frame. For a Newtonian 2-body system, the Keplerian orbit does not rotate in space; one simply cannot get a rotating ellipse using Newtonian, twobody equations of motion. For such a system, the perihelion direction acts as an inertial pointer; the period of an orbit, measured between successive perihelion passages, is determined with respect to inertial space. Similarly, for the real solar system, the small rotations of an elliptical orbit are due to relativistic effects and to gravitational perturbations from the other bodies of the system. However, these effects are well-known and well-modeled.

Correct gravitational physics, along with the observational data, demands an inertial reference frame in order for the observations to be fit properly. If the observational residuals contain systematic, non-inertial errors, it is not the fault of the frame; the problem lies with the observations themselves or with their reductions. If the orbit fits the observations well, then the ephemeris is accurate in inertial space, mean motion and all.

Even with fitting to only optical data, an ephemeris adjusts itself onto an inertial frame. The period of a planet can be determined and the motion of the perihelion constrained by physics; similarly, the motion of the orbital plane is determined by physics. Thus, a rotation in a catalog will introduce inconsistencies between the physical model and the observations, albeit with limited accuracies. Previously, uncertainties in precession, catalog proper motions, etc., determined from optical observations alone, were on the order of $1^{\prime \prime} /$ cty.

Modern ephemerides of the inner planets, Moon, and Sun are now fit to highly accurate ranging observations: radar (uncertainties of 100-200 meters, spanning 3 decades), lunar-laser-ranging (now at the 2-3 cm level), and spacecraft-ranging (10 meter accuracy). See Standish et al. (1995) and Standish (1998) for more details about the observations. Fitting to these observations determines all inner planet mutual distances and angles to an accuracy of about 1-2 kilometers (1-2 milliarcseconds), and it determines the mean motions with respect to inertial space to the level of about $0.01 /$ cty (equivalent to about 10 
kilometers per century). Williams and Standish (1989) describe how well various orbital elements are determined by the fitting process and provide estimates of the uncertainties.

Importantly, there is one part of the ephemerides that is not provided by fitting to ranging observations: the orientation of the whole system onto an external reference frame. Fortunately, this orientation is now provided by ICRFbased VLBI observations of the Magellan spacecraft in orbit about Venus and the Phobos spacecraft on its approach to Mars. These observations are discussed next.

\section{VLBI of Spacecraft near Planets}

VLBI measurements of a spacecraft with respect to the ICRF radio sources have been combined with a planet-centered spacecraft trajectory in order to yield a positional determination of the planet. Fitting to these observations automatically orients the ephemeris onto the ICRF, and since ranging observations tightly connect all of the inner solar system ephemerides, it is the full inner planet system that is oriented onto the ICRF.

A VLBI observation, taken over a relatively short time-span, is essentially a one-dimensional measurement. For observations taken between Goldstone and Madrid, where the latitudes of the two antennas are roughly the same, the determination is almost completely in right ascension; the corresponding uncertainty ellipse, very long and narrow, is oriented nearly vertically in an $\alpha-\delta$ plot. I.e., the uncertainty lies along the $\delta$-axis: $\alpha$ is well-determined; $\delta$ is not. For observations between Goldstone and Canberra, the ellipse is tilted at about $45^{\circ}$ from vertical, so that the determination is a linear function between $\alpha$ and $\delta$.

The four plots in Figure 1 illustrate four VLBI observations. In each, the $1 \sigma$ uncertainty ellipse is shown; the one-dimensional determination, itself, is the loci of points midway between the two lines of the uncertainty ellipse; the distance from this line to the origin represents the minimum residual of the possible combinations of $\Delta \alpha$ and $\Delta \delta$. In the upper left figure, the observation of Magellan at Venus was evidently taken between Goldstone and Madrid, showing roughly, $\Delta \alpha \approx-0$ "'003 \pm 0 "'002. The upper right figure, between Goldstone and Canberra, indicates a linear combination of $\alpha$ and $\delta$. The observations in the two lower figures are from Phobos at Mars; the wider lines of these two ellipses indicate the higher uncertainties for the Phobos observations.

Table 1 lists all of the VLBI observations for Venus and Mars. The angle, $\theta$, gives the direction of the determination from the origin $90^{\circ}$ from the uncertainty ellipse). The distance, $\nu$, is measured from the origin in the direction of $\theta$; it gives the distance of the observation line from the origin.

It is evident from the table that the full set of observations determines the orientation of the ephemerides with an uncertainty of about \pm 0 ". 001 . For typical distances of the inner solar system, this is equivalent to an uncertainty on the order of 1 kilometer. 
DE405: Magellon at Venus 1994 AUG 02

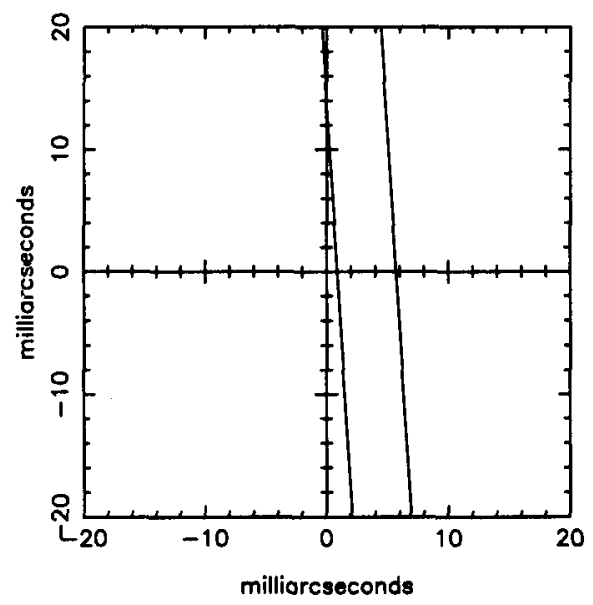

DE405: Phobos at Mars 1989 FEB 17

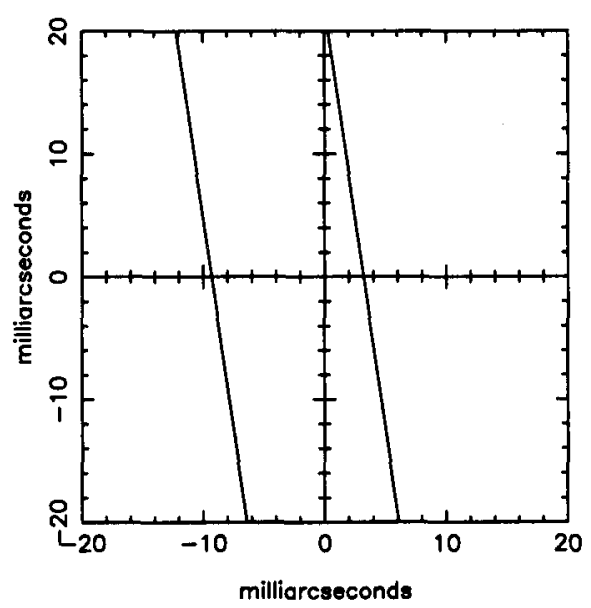

DE405: Mogellan at Venus 1994 AUG 10

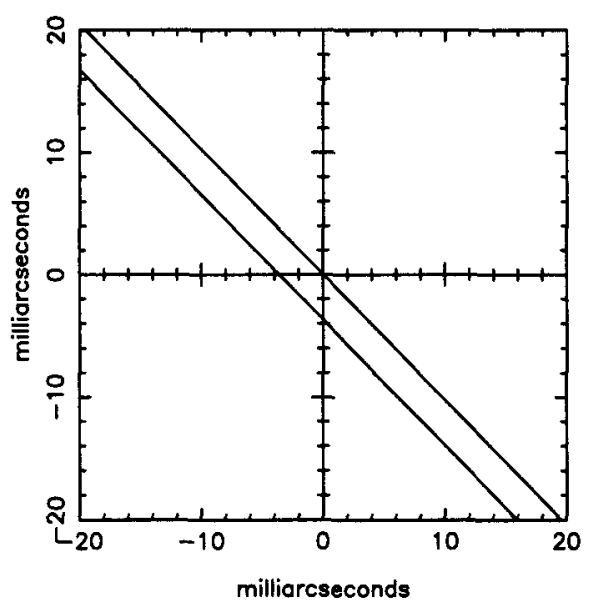

DE405: Phobos ot Mars 1989 MAR 25

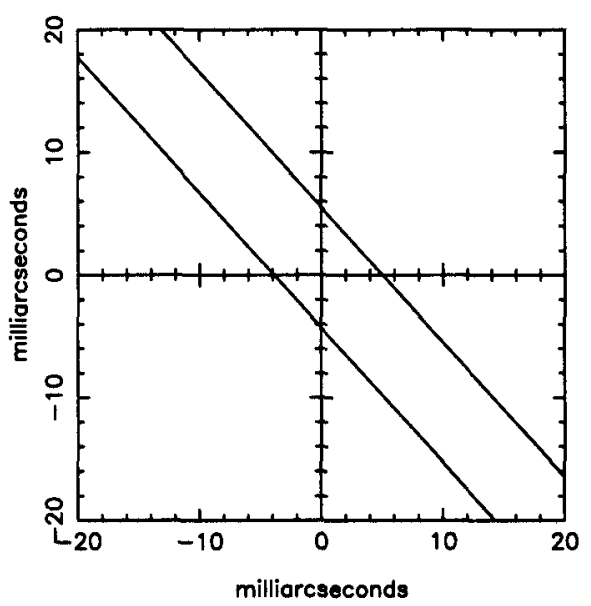

Figure 1. VLBI observations of spacecraft near to Venus and Mars. Four illustrative examples are shown; see text for explanation. 
Table 1. VLBI observations of spacecraft near to Venus and Mars. The angle, $\theta$, gives the orientation of the observation (see the text); the residual, $\nu$, its uncertainty, $\sigma$, and their ratio are given. It is evident that the resultant orientation of the inner solar system ephemerides is accurate to about \pm 0 !. 001 .

\begin{tabular}{clrrr} 
& & $\theta$ & $\nu \pm \sigma$ & $|\nu| / \sigma$ \\
Venus & \multicolumn{1}{c}{$\begin{array}{c}c \\
\text { 1990 Sep 12 }\end{array}$} & 45.6 & $-2.4 \pm 1.0$ & 2.40 \\
& 1991 Dec 22 & 43.2 & $0.4 \pm 2.0$ & 0.20 \\
1991 Dec 23 & 41.9 & $-2.6 \pm 2.9$ & 0.88 \\
1992 Mar 29 & 45.0 & $1.0 \pm 1.8$ & 0.53 \\
1994 Apr 01 & 3.1 & $-1.7 \pm 1.8$ & 0.92 \\
1993 Nov 10 & 48.8 & $0.2 \pm 1.1$ & 0.22 \\
1994 Feb 03 & 46.0 & $3.7 \pm 2.0$ & 1.85 \\
1994 Mar 07 & 44.4 & $1.4 \pm 2.2$ & 0.64 \\
1994 May 21 & -6.2 & $-2.2 \pm 1.9$ & 1.15 \\
1994 May 22 & 49.9 & $7.5 \pm 2.5$ & 2.97 \\
1994 May 28 & 53.2 & $6.6 \pm 2.1$ & 3.06 \\
1994 Jun 25 & -1.0 & $1.5 \pm 2.3$ & 0.68 \\
1994 Jun 26 & 52.3 & $1.2 \pm 1.9$ & 0.63 \\
1994 Jul 08 & 2.7 & $3.9 \pm 2.6$ & 1.49 \\
1994 Jul 09 & 46.0 & $3.0 \pm 3.2$ & 0.92 \\
1994 Jul 21 & 3.4 & $2.6 \pm 4.0$ & 0.64 \\
1994 Aug 02 & 3.5 & $3.3 \pm 2.4$ & 1.36 \\
1994 Aug 10 & 44.4 & $-1.3 \pm 1.3$ & 0.98 \\
1989 Feb 17 & 8.2 & $-3.0 \pm 6.2$ & 0.49 \\
Mars & & & &
\end{tabular}




\section{Ephemeris Uncertainties}

As mentioned earlier, the major part of the uncertainties of the inner planet ephemerides is due directly to the uncertainties in the masses of the asteroids which perturb the inner planets. For the JPL ephemerides, the asteroids, 0001 Ceres, 0002 Pallas, 0004 Vesta, 0007 Iris, and 0324 Bamberga are handled separately. They are each assigned a mass and their perturbations upon each of the major planets is included in the equations of motion of the integration program. Perturbation effects for 295 other asteroids are included, using masses computed from each asteroid's estimated diameter and using a density according to the asteroid's taxonomic class.

The asteroid's perturbations disturb the orbital motions of Mars and the Earth. Uncertainties in the asteroids' densities and volumes lead to uncertainties in the masses, sometimes by as much as a factor of two. Consider covariances, Monte Carlo experiments using actual integrations, and sensitivity studies - all give approximately the same results: the uncertainties in the relative positions and motions of the inner planet ephemerides, introduced from the uncertainties of the asteroid masses, are on the order of 1 kilometer. These uncertainty estimates are also in agreement with those of Williams (1994) in his discussion of the asteroids' effects on ranging to Mars. It is coincidental that the uncertainties from the asteroid masses are the same size as the uncertainties from orienting the ephemerides onto the ICRF using VLBI.

It should be mentioned, as an aside that the uncertainties for the Moon are due to something else entirely. They come from tidally-induced deceleration in longitude which has an uncertainty of about $0.5 / \mathrm{cty}^{2}$.

\section{Future Improvement to the Ephemerides}

Accurate ephemerides for future dates must rely on one of two things: either the ephemerides must be updated continuously with current, accurate observational data, or there must be some significant improvement in the knowledge of asteroid masses. For the latter, there are two evident methods: either the masses of many asteroids must be determined individually, or the method for estimating a mass for a given asteroid must be improved. This latter requires improvements in the estimation of asteroid volumes, improvements in the measuring of taxonomic classes, and improved understanding of how to assign a density given the taxonomic class.

\section{Conclusions}

There still is a dynamical reference frame, as in the past. It is still defined by the planetary and lunar ephemerides. Now, however, it is made to be coincident with the ICRF. The ICRF is assumed to be inertial; certainly, at least, any non-inertial motion of the ICRF is far less than that of the dynamical reference frame. As a result, the use of the dynamical frame for the determination of inertial space is no longer an integral part of astronomical reference frames.

Ephemeris creation now depends upon two major types of observations: ranging and VLBI. For the data reductions, it is necessary to locate the observ- 
ing stations using ICRF-based Earth orientation parameters from the IERS. Since all three reference frames involved in the process (ephemeris, VLBI, Earth orientation) are now based upon the ICRF, there is no longer an explicit use of the celestial equator, equinox, or ecliptic in the ephemeris creation process. These elements are obsolete in that context. Of course, it should be stated that the reduction of older observational data, still useful for the outer five planets, does indeed rely upon such elements.

The accuracy of present ephemerides is limited primarily by the uncertainties in the masses of the asteroids. Future improvements to the ephemerides must await significant progress in the determination of asteroid masses.

Acknowledgments. The research in this paper was carried out at the Jet Propulsion Laboratory, California Institute of Technology, under a contract with the National Aeronautics and Space Administration.

\section{References}

Standish, E. M., Newhall, X X, Williams, J. G. and Folkner, W. M., 1995, "JPL Planetary and Lunar Ephemerides, DE403/LE403," JPL IOM 314.10127.

Standish, E. M., 1998, "JPL Planetary and Lunar Ephemerides, DE405/LE405," JPL IOM 312.F-98-048.

Williams, J. G., 1984, "Determining Asteroid Masses from Perturbations on Mars," Icarus, 57, 1-13.

Williams, J. G. and Standish, E. M., 1989, "Dynamical Reference Frames in the Planetary and Earth-Moon Systems", in Reference Frames (J. Kovalevsky, I. I. Mueller and B. Kolaczek, eds.) Kluwer Academic Publishers, Dordrecht. 